\title{
Advance gait analysis in clinical assessment
}

\begin{abstract}
Advanced three dimensions (3D) gait analysis provided the quantitative information and objective data needed to identify and analyze walking and posture problems, load anomalies and muscle failure, which would not be measurable with normal clinical exams. The digital synchronization of different analysis tools helps to simultaneously compare, frame by frame, the patient's movements of limbs and muscles and force distribution on the surface during movement. The core of gait analysis is the measurement of joint kinematics and kinetics. Other measurements regularly made are dynamic electromyography (EMG), oxygen consumption and foot pressures. The examples of advanced gait analysis in clinical assessment are also included in this mini review.
\end{abstract}

Volume 2 Issue 2 - 2017

\author{
Areerat Suputtitada \\ Department of Rehabilitation Medicine, Chulalongkorn \\ University, Thailand
}
Correspondence: Areerat Suputtitada, Department of Rehabilitation Medicine, Faculty of Medicine, Chulalongkorn University, Thailand, Email prof.areerat@gmail.com, areerat.su@chula.ac.th

Received: August 20, 2017 | Published: November 15, 2017

Keywords: gait analysis, clinical assessment, kinematics, kinetics, dynamic electromyography (EMG)

\section{Introduction}

The simple form of gait assessment is practiced every day in orthopedic rehabilitation and neurorehabilitation. To perform a formal gait assessment requires a careful examination of the gait, using systematic approach. Quantitative gait analysis can precisely and objectively characterize specific aspects of walking. Joint motions and external moments can be calculated from the positions of reflective markers on body segments and ground reaction forces recorded during walking. External moments must be balanced by internal moments produced by the muscles and other joint structures. Different types of gait analysis data may be useful for different aspects of gait assessment. Information of the foot timing may be useful to identify asymmetries and may indicate problems with balance and stability. The core of most contemporary gait analysis is the measurement of joint kinematics and kinetics. Other measurements regularly made are electromyography (EMG), oxygen consumption and foot pressures. A systematic physical examination of the patient is usually conducted as part of a gait analysis. Clinical gait analysis is performed to allow the selection from amongst treatment options (including the possibility of not intervening). This is based on one or more of:

i. Diagnosis between disease entities.

ii. Assessment of the severity, extent or nature of a disease or injury.

iii. Monitoring progress in the presence or absence of intervention.

iv. Prediction of the outcome of intervention (or the absence of intervention).

Passive-reflective markers were placed on the skin in relation to bony landmarks. These were illuminated markers and detected by modified video cameras. If two or more cameras detect a marker and the position and orientation of these cameras are known then it is possible to detect the three-dimensional position of that marker. Whilst the basic principles remain the same as the earliest systems, the speed, accuracy and reliability has advanced beyond all recognition. It is now common to find clinical systems using 8,10 or more cameras functioning at over $100 \mathrm{~Hz}$ and capable of detecting reliably the presence of many tens of markers of between 9 and $25 \mathrm{~mm}$ diameter. Calibration of the systems (the determination of the position, orientation and optical and electronic characteristics of the cameras) can generally be accomplished in less than a minute. Marker positions from clinical trials can be reconstructed and markers labelled automatically in real time (although this feature is often not essential for clinical studies). The determination of the accuracy of such systems is now generally limited by the accuracy of any alternative means to determine marker position and can be taken to be of the order of $1 \mathrm{~mm}$.

Gait analysis interpretation is involving the following steps

i. Joint angle: what is the angle of a joint at a particular part of the gait cycle and in which direction is it moving?

ii. Joint moment is the internal moment extensor or flexor? This will indicate which muscles or ligaments are under tension and to what degree

iii. Joint power: is power generation or absorption taking place? This would indicate concentric or eccentric contraction, or storage and release of energy by stretching elastic tissues

iv. EMG: is the muscle electrical activity consistent with the kinematics data?

\section{Example of gait analysis in clinical assessment}

An effect of side-of-lesion was revealed in average peak lateral ground reaction force data. Larger lateral ground reaction forces acting on the left non paretic stance limb of right paretic patients compared to the right non paretic stance limb of left paretic patients during gait initiation may be an indication of differing adaptations that depend on the side-of-lesion. ${ }^{1}$ More significant differences from normal were found in symptomatic leg length inequality patients than in asymptomatic total hip replacement patients. The leg length inequality patients had altered functions defined by lower gait velocity, reduced stride length, reduced ground reaction force, decreased hip range of motion, reduced hip moment and less dynamic hip force with a 24\% lower heel-strike peak, $66 \%$ higher mid-stance trough and $37 \%$ lower toe-off peak. Greater asymmetry in hip contact force was also observed in leg length inequality patients. ${ }^{2}$

The walking velocity and stride length were reduced significantly in the postural instability and gait disturbance (PIGD) group compared to the tremor-dominant (TD) group. The PIGD group 
showed a significantly reduced range of motion in the pelvic and lower extremity joints by kinematics. Stride time variability was significantly increased and the pelvic oblique range was significantly reduced in the freezing of gait (FOG) group. There are differences in the perturbation of the basal ganglia-cortical circuits based on major clinical features. The reduction of the pelvic oblique range of motion may be a compensatory mechanism for postural instability and contributes to stride time variability in patients with FOG. ${ }^{3}$

There were statistically significant differences in $3 \mathrm{D}$ gait analysis between healthy elderly group and the mild Parkinson (PD) group, between the mild and moderate PD group in stride velocity, stride length, and in the hip and ankle joints kinematics data: on initial contact, on maximum extension during terminal contact and on maximum flexion during mid-swing. The clinical instruments had significant correlation with in stride velocity and stride length. The novel of 3D gait analysis significantly characterized the difference between mild PD and normal healthy elderly, mild PD and moderate PD. The 3D gait analysis can be used to confirm clinical diagnosis of early Parkinson's and differentiate from elderly. ${ }^{4}$

The gait variables most sensitive in giving evidence of a change in a patient's ambulatory status were maximal vertical force and average velocity followed by step rate, single limb support, and weight acceptance - all measurements of weight bearing capacity. Maximal vertical force and average velocity were the only gait variables that could distinguish before and after total hip replacement, after total knee replacement (THR) and in comparison of two different methods for THR, one cemented (Charnley) and one non-cemented (HP Garches).In correlations between the clinical impression of gait function and its objective registration the strongest correlations were found in maximal vertical force and average velocity. Thus, gait analysis is considered a useful tool in the evaluation of loco motor function after surgical procedures. ${ }^{5}$

The knee kinematics and kinetics of Grade II knee osteoarthritis (OA) were measured during level walking, stair ascent and stair descent. The patients with knee OA had a significantly reduced internal knee extensor moment compared to normal subjects. This difference reflects the patient's compensation to reduce the knee joint loading. Further, subjects with OA and a higher body mass index have a lower knee extensor moment. The female subjects had significantly greater knee flexion and a greater knee extensor moment. This gender difference may partially explain the increased prevalence of $\mathrm{OA}$ in females. Most tests of OA treatments are assessed by criteria that do not reflect functional activities. This study demonstrates that objective gait analysis can be used to document gait adaptations used by patients with knee OA. ${ }^{6}$

Biomechanical variables, associated with Osteoarthritis (OA) severity such as external knee adduction moment (KAM) and joint malalignment, may affect the disease process by altering the boneon-bone forces during gait. The magnitude and time course of KAM during gait appeared to be consistent across laboratories and computational methods. A higher peak KAM for the OA group when compare to normal group.. Knee adduction moment increased with $\mathrm{OA}$ severity and was directly proportional to varus malalignment. Classifying the patients on the basis of disease severity decreased the group variability, permitting the differences to be more detectable. Biomechanical variables such as varus malalignment are associated with KAM and therefore may affect the disease process. These variables should be taken into considerations when developing therapeutic interventions for individuals suffering from knee OA. ${ }^{7}$

External ankle support has been successfully used to prevent ankle sprains. In order to investigate the effect of external ankle support (braces and high-top shoes) on the ankle and knee joint loading during a netball specific landing task. The kinematic and kinetic data were collected simultaneously using a3-D Motion Analysis System and one Kistler force plate to measure ground reaction forces. Players performed a single leg landing whilst receiving a pass while wearing a standard netball shoe, the same shoe with a lace-up brace and a high-top shoe. Only the brace condition significantly reduced the ankle range of motion in the frontal plane (in/eversion) by $3.95 \pm 3.74$ degrees compared to the standard condition. No changes were found for the knee joint loading in the brace condition. The high-top shoes acted to increase the peak knee internal rotation moment by $15 \%$. Both the brace and high-top conditions brought about increases in the peak ankle plantar flexion moment during the landing phase. Lace-up braces can be used by netball players to restrict ankle range of motion during a single leg landing while receiving a pass without increasing the load on the knee joint. ${ }^{8}$

Footwear modifications have been investigated as conservative interventions to decrease peak external knee adduction moment (EKAM) and pain associated with knee osteoarthritis (OA). Seventeen studies included healthy individuals and 19 studies included subjects with medial knee OA. Quality assessment (modified Downs and Black quality index) showed an (average \pm SD) of $73.1 \pm 10.1 \%$. The most commonly used orthotic was the lateral wedge, with three studies on the medial wedge. Lateral wedging was associated with decreased peak EKAM in healthy participants and participants with medial knee OA while there is evidence for increased peak EKAM with the use of medial wedges. Modern footwear (subjects' own shoe, "stability" and "mobility" shoes, clogs) were likely to increase the EKAM compared to barefoot walking in individuals with medial knee OA. Walking in innovative shoes ("variable stiffness") decreased the EKAM compared to control shoes. Similarly, shoes with higher heels, sneakers and dress shoes increased EKAM in healthy individuals compared to barefoot walking.Further development may be needed toward optimal footwear for patients with medial knee OA with the aim of obtaining similar knee moments to barefoot walking. ${ }^{9}$ In conclusion, advanced gait analysis provided the quantitative information and objective data needed to identify and analyze gait and motion problems. The data are essential for proper management in complicated patients in addition to the clinical assessment.

\section{Acknowledgements}

None.

\section{Conflict of interest}

Professor Dr. Areerat Suputtitada declares no conflict of interest.

\section{References}

1. Sharma S, McMorland AJ, Stinear JW. Stance limb ground reaction forces in high functioning stroke and healthy subjects during gait initiation. Clin Biomech. 2015;30(7):689-695.

2. Li J, McWilliams AB, Jin Z, et al. Unilateral total hip replacement patients with symptomatic leg length inequality have abnormal hip biomechanics during walking. Clin Biomech. 2015;30(5):513-519. 
3. Koh SB, Park KW, Lee DH, et al, Gait Analysis in Patients With Parkinson's Disease: Relationship to Clinical Features and Freezing. $J$ Mov Disord. 2008;1(2):59-64.

4. Suputtitada A, Sanguanrungsirikul S. Poster 4: 3D Gait Analysis in Parkinson's and Elderly: What are the Differences? Arch Phys Med Rehabil. 2013;94(10):e13.

5. Olsson E. Gait analysis in hip and knee surgery. Scand J Rehabil Med. 1986;Suppl 15:1-55.

6. Kaufman KR, Hughes C, Morrey BF, et al. Gait characteristics of patients with knee osteoarthritis. J Biomech. 2001;34(7):907-915.
7. Foroughi N, Smith R, Vanwanseele B. The association of external knee adduction moment with biomechanical variables in osteoarthritis: a systematic review. Knee. 2009;16(5):303-309.

8. Vanwanseele B, Stuelcken M, Greene A, et al. The effect of external ankle support on knee and ankle joint movement and loading in netball players. J Sci Med Sport. 2014;17(5):511-555.

9. Radzimski AO, Mündermann A, Sole G. Effect of footwear on the external knee adduction moment - A systematic review. Knee. 2012;19(3):163-175. 